\title{
Determining the Ideal Initial Printing Colorants in Electrophotography by the Discrete Gradation Trajectories
}

\author{
Dmitry TARASOV ${ }^{1}$ and Oleg Milder ${ }^{1}$ \\ ${ }^{1}$ Ural Federal University named after the first President of Russia B N Yeltsin
}

September 21, 2020

\begin{abstract}
The accuracy and repeatability of the color reproduction in print is determined by the fine tuning of the tone reproduction curves of the basic printing colorants (most often this is CMYK). However, the diversity of manufacturers of printing equipment and dyes introduces an element of significant uncertainty about color uniformity. In addition, the traditional approach does not take into account the effect of hue change when applying the original dyes, as well as, the non-linearity of the hue rise in high and low density areas. Determining the color of base colorants that produces the most uniform tone change is an important engineering challenge. Previously, there was no scientific basis for such calculations. We recently proposed an alternative color correction model based on gradation trajectories as an analogue of gradation curves in the CIE Lab space. We have also described the extension of the approach to double color overlay (gradation surfaces) and its analytical and discrete implications. The trajectories are the geodetic lines on gradation surfaces. In this paper, we propose using the gradation trajectories to determine "ideal" or "true" initial printing dyes for electrophotography. To simplify calculations, natural color discretization in digital printing is used.
\end{abstract}

\section{Introduction}

In the color management, the color reproduction adjustment is closely related to the fine tuning of the basic colorants gradations. This approach works in conjunction with various color prediction models embedded in the color management systems and aims to adjust colors, save amount of inks used, and to predict the resulting color in print. Initial colorants in the frame of the standard subtractive color model are represented by three basic colors (CMY) and one additional Black (K). The color formation in such a model is a separate complicated task, although all color prediction models have to rely on those four colorants and their overlapping.

On the other hand, a few is known about the initial colorants in real printing systems. Usually, each manufacturer uses its own recipe of the CMYK inks formation based on the fossil pigments. Hence, each color reproduction system, in fact, uses a unique initial colorants set, especially, considering the non-ideality of the printing system itself. Anyway, each system reproduces quite accurate colors after the procedure of calibration and color tuning with the help of color prediction models used in the color management software is carried out.

There are many different color prediction models applied in color management. The empirical surface models take into account superposition of ink halftones and do not deal with the light propagation and fading within the print. The physically inspired models engage a more detailed analysis of light-print interaction based on the prediction of how the light paths go within a halftone print and what the resulting fade is. The ink spreading models characterize the effective surface of an ink dot after it has been printed at a given nominal 
surface coverage compared to the effective surface coverage that forms the physical dot gain. The spectral reflection color prediction models deal with spread-based light propagation-transportation probability. These models study the impact of different factors influencing the range of printable colors (the inks, substrate, illumination conditions, and halftones) and create the printer characterization profiles for the purpose of color management [1]. These models together with the ink-spreading models take into account the physical dot gain and are able to predict the reflectance spectra as a function of ink surface coverage for $2-4$ inks (binary and ternary color systems). The models use the multiple tone reproduction (ink spreading) curves to characterize the physical dot gain of the ink halftones on the substrate and under all solid ink-superposition conditions $[2-4]$.

The color prediction models have been successfully applied to the color reproduction management [5-9], however, all the proposed approaches require a significant number of measurements, computations and checks. However, since most of them are based on empirical relationships, the accuracy of predicting some shades of the reflection spectrum remains low.

If one looks at the problem of color reproduction from the other side, the use of the "ideal" initial colorants might be an excellent solution providing the basic color stability. An ideal colorant is such a combination of the initial colorants, which ensures minimal fluctuations of color tone in the range from a white substrate to a full dye. In this case, the ideal colorant will be a specific mixture of two initial colorants.

In previous works, the gradation trajectories (GT) as a three-dimensional interpretation of tone reproduction curves (TRC) in CIE Lab space were introduced [10-12] (see also Fig.1 (a, b)). Thus, any color is uniquely described by a three-component vector in the 3D Lab space. The metric of the space is the color difference $d E$ (or $\Delta E$ ), which is defined at least by the root squared sum of color components differences. Presence of any metric in the sense of the method of measuring distances turns the color space into a metric one.

FIGURE 1. (a) CIE Lab color space body, (b) gradation trajectory with projections, (c) gradation surface with experimental dots.

The GT's arc length is defined as an integral of the $d E$ units. The arc is split into $n$ segments of equal length in order to match them with the same tone increment. We have suggested an empirical description of the GT by polynomials of the $4^{\text {th }}$ degree, as well as the discrete approach to the GT computation on the basis of intrinsic color quantization in the digital printing systems. Further, we expanded the approach onto the binary overlapping of the initial colorants. Thus, the method of gradation surfaces (GS) has been developed [13]. On the basis of the proposed approaches, methods were developed for determining the linearization of various printing systems and determining the gray balance [14]. In the case of printing colorants, their paired double overlays (binaries) correspond to additive primary colors (RGB). Since the RGB and CMYK spaces are both device-dependent, there has not been any simple or general transformation formula that converts them. Using the gradation surface as a gradation trajectory of two colorants, we have suggested the way to develop such a conversion.

If we consider an ideal colorant's GT as a particular geodesic line on the GS of some basic colorants, then we could calculate it using both an analytical and discrete approaches. This work aims to establish a discrete approach for searching the ideal colorants' gradation trajectories based on a color quantization in digital printing systems.

\section{APPROACH and experiment}

Notions of gradation trajectories and gradation surface remain the same. The gradation trajectory is a geometric place of the points in the CIE $L a b$ space, which coordinates correspond to the CIE Labcoordinates of the individual patches of the initial colorants $(\mathrm{C}, \mathrm{M}, \mathrm{Y}, \mathrm{K})$. We assume that the tone part in the layout is denoted as a tparameter in the range from zero (paper) to one (full dye). Therefore, the trajectories might be analytically described by polynomials of $n^{\text {th }}$ degree (1), since color changes continuously in accordance with 
continuous increment of $t$. The gradation surface of double colorants overlapping is a surface "stretched" on two gradation trajectories of the initial colorants. Extending the concept of gradation trajectories on the two-dimensional case, we define the gradation surface as a locus of points in the CIELab space where coordinates correspond to the CIE Labcoordinates of the individual patches of the binary halftone scale from zero (unprinted substrate) to two (full dye of 2 colorants). By analogy with the gradation trajectory, the gradation surface is also expressed by a polynomial of $n^{\text {th }}$ degree (2).

\{

$$
\begin{gathered}
t \in[0 ; 1] \\
a=a_{4} t^{4}+a_{3} t^{3}+a_{3} t^{3}+a_{1} t+a_{0}, \\
b=b_{4} t^{4}+b_{3} t^{3}+b_{3} t^{3}+b_{1} t+b_{0} \\
L=\left(L_{0}-L_{\infty}\right) \times \exp \left(-L_{3} t^{3}-L_{3} t^{3}-L_{1} t\right)+L_{\infty},
\end{gathered}
$$

\{

$$
\begin{gathered}
\overline{m, n} \in[0 ; 1] \\
a(m, n)=a_{0}+\sum_{i=1}^{4} \sum_{j=0}^{i} a_{i-j, j} \bullet m^{i-j} \bullet n^{j}, \\
b(m, n)=b_{0}+\sum_{i=1}^{4} \sum_{j=0}^{i} b_{i-j, j} \bullet m^{i-j} \bullet n^{j}, \\
L(m, n)=\left(L_{0}-L_{\infty}\right) \exp \left(-\sum_{i=1}^{3} \sum_{j=0}^{i} L_{i-j, j} \bullet m^{i-j} \bullet n^{j}\right)+L_{\infty},
\end{gathered}
$$

where $L_{i}, a_{i}, b_{i}$ are some numerical coefficients, $L_{0}, a_{0}, b_{0}$ are the CIE $L a b$ coordinates of the unprinted substrate, $L_{\infty}$ is the visual brightness of a hypothetical continuous ink layer of infinite thickness, $t$ is the receipt of the tone in the layout, $(m, n)$ is the receipt of the binary overlap (in parts, percent or color "quanta").

Color quantization in digital printing system with the color depth 8 bit per color channel is accounted as a virtual grid of $2^{8}=256$ lines per 1 channel and $2^{16}=65536$ possible halftones of a chosen pair of colorants. The halftone recipes achievable within a given printing system are in the virtual grid nodes. Each reproducible tone has its own recipe $(m, n)$ and a set of the CIE $L a b$ coordinates.

Introducing the color quanta based on the above reasoning, we have to start from the fact that the tone fraction must take values from a particular discrete series. Thus, to account the color quantization in a real print system, the real tone fraction values should be transformed according to the formula

$t_{\text {fact }}=\frac{\text { uint } 8\left(t_{\text {des }} \times 255\right)}{255},()$

where $t_{\text {des }}$ is the desired tone fraction, $t_{\text {fact }}$ is the actually realizable tone fraction, uint 8 is the Matlab function for rounding to an integer in the range 0-255 (8-bit integer). Thus, each patch recipe component $(m, n)$ is rounded to the 8-bit integer that forms a grid of the color quanta replacing the initial colorant percentage.

We showed that the best trajectory of a binary surface is the geodesic on a gradation surface between $(0,0)$ and $(1,1)$ points. Hence, we will find the gradation trajectory of an ideal colorant assuming that, first, it exists and is unique, second, it is a geodesic of two adjacent colorants. The criterion for determining such a trajectory is the minimum color difference $d E 2000$ between each successive pair of the color quanta.

For the experiment, we have selected the electrophotographic Konica-Minolta Bizhub Pro C6000L as a printing system with the print resolution $1200 \times 1200$ dpi and the RIP EFI Fiery IC-306. Paper substrate: Moorim Neo Star Matt coated, $140 \mathrm{~g} / \mathrm{m}^{2}$. The test gradation scales were synthesized using the ChartGenerator in theMeasureTool in the ProfileMaker package for the X-Rite i1 iSis automatic spectrophotometer. The test chart contained 2115 gradation patches that correspond to the 15-quanta tone increment for each colorant forming a binary surface. The experiment algorithm is shown in Fig. 2.

Gradation scales with an approximately $5 \%$ increment of the tone in the layout from 0 to $200 \%$ were implemented. Since the tone fraction must take values from the discrete series $[0 ; 0.05 ; 0.1 \ldots 0.95 ; 1]$, the fraction values should be transformed according to the discretization formula (3). The numerator of formula 
(3) is the expression of color tone in each color quanta. The expression of the color tone in quanta is initially formed in terms of the bit depth of the printing system and is preferable to use. Whichever way of specifying a color is chosen, formula (3) allows establishing an unambiguous relationship between them.

FIGURE 2. The experiment algorithm.

The algorithm used for the sequential quantized recalculation takes place since each of the components of the gradient surface of the color difference increment with respect to the unprinted substrate changes sign at least only once. In other words, the surface of color differences to the substrate color has no local maxima or minima.

For each point of the isoline, the sum of the color differences to the point corresponding to the initial (for instance, unprinted paper) and target points (for instance, full dye Cyan) is computed. Among all these sums corresponding to one $p$, the lowest value has been found. The recipe $(m, n)$ corresponding to this minimal sum at the isoline defines the recipe of the geodesic crossing this isoline. The complete set of points found in this way forms the desired geodesic. This line provides the shortest path from the paper point to the point of complete double overlap. This is the major result of the proposed algorithm.

\section{REsults and discussion}

The results of the sequential execution of the algorithm for each gradation surface (RGB) and two initial colorants (CMY) depicting the projections of the ideal colorants on the corresponding surfaces are shown in Fig. 3. At the first look, these projections should complement each other in areas where the geodesic of one surface fits its boundary. As follows from Fig. 3, it is not so. This can mean only one thing: the ideal colorant does not lie on any of the surfaces of double overlays but is inside the body of the color gamut of the printing system. In other words, more than two initial colorants that are necessary for its formation.

The projections of the ideal Magenta on the Blue and Red surfaces are of particular interest. The broken character of the projections is related to the fact that the localization area of the ideal Magenta is close to the singularity points of the formula $d E_{2000}$. In the case of electrophotography, it is obvious that modeling of the ideal colorants is at the moment rather speculative. The real implementation of toners with the "ideal" colors for use in printing systems is impossible for reasons that formed the basis of the Yule-Nielson model. When hypothetically creating these ideal colorants, it will be necessary to completely revise the model of subtractive color synthesis.

FIGURE 3. Geodesics as the projections of the ideal colorants on the corresponding gradation surfaces

\section{Conclusion}

The work is aimed to create an approach to the ideal colorants definition and calculation. We assume the existing of the relationship between two device-dependent color spaces (CMYK and RGB) accounting the color quantization in print (as for instance, in electrophotography). This makes the systems to be able to precisely express primaries of additive color synthesis (R, G, B) as binaries of subtractive color synthesis colorants (C, M, Y). For this, the universal CIE Labcolor space is used in computations. The binary surface is defined as a gradation surface of two colorants that is "stretched" on their gradation trajectories.

An ideal colorant is such a combination (mixture) of the initial colorants, which ensures the minimal fluctuations of color tone (i.e. color difference) in a range from an unprinted substrate to a full dye. The gradation trajectory of the ideal colorant is the geodesic on the gradation surface of two adjacent initial colorants. The trajectory satisfies the condition of the minimum possible color difference between the color quanta that form it. A discrete interpretation of the ideal colorant computation is proposed in terms of gradation trajectories in the CIE $L a b$ space together with the color quanta as an expression of color quantization. 
In the case of electrophotography, the practical implementation of the ideal printed colorant will require a complete revision of the model of subtractive color synthesis, which is hardly in the interests of production. However, this question is extremely interesting from a theoretical point of view. The case of inkjet printing, also, requires particular consideration.

\section{References}

1. R. Bala, "Device characterization" in Digital Color Imaging Handbook, edited by G. Sharma (CRC Press, Boca Raton, FL, 2003), pp.269-379.

2. R. Balasubramanian, J Elec. Imag. 8 , 156-166 (1999).

3. R.D. Hersch and F. Crété, "Improving the Yule-Nielsen modified spectral Neugebauer model by dot surface coverages depending on the ink superposition conditions" in Proc. SPIE 5667 (2005), pp. 434445.

4. D.R. Wyble and R.S. Berns, Col.Res.\&App. 25 , 4-19 (2000).

5. N.P. Garg, A.K. Singla, and R.D. Hersch, J.Imag.Sci.Tech52 (4), 040908-040908-5 (2008).

6. J.S. Arney, P.G. Engeldrum, and H. Zeng, J.Imag.Sci.Tech 39, 502-508 (1995).

7. S. Livens, "Optimisation of Printer Calibration in the Case of Multi Density Inks" in Conference on Color in Graphics, Imaging, and Vision, CGIV 2002. Final Program and Proceedings (2002), pp. 633-638.

8. L. Chagas, A. Blayo, and P. Giraud "Color Profile: methodology and influence on the performance of ink-jet color reproduction" in IS\&T's NIP20. International Conference on Digital Printing Technologies (2004), pp. 655-659.

9. Y-J. Wu Reducing "Ink-jet Ink Consumption with RIP software for POP Display Media. Digital Fabrication and Digital Printing" in NIP30 Technical Program and Proceedings (2014), pp. 108-111.

10. M. Titova, O. Milder, and D. Tarasov "Gradation trajectories: acquisition and analysis" in Proceedings of the Conference «Information: transmission, processing, perception $\gg$. UrFU (2016) pp. 120-125 (in Russian).

11. O.B. Milder, D.A. Tarasov, and M.Yu Titova "Inkjet Printers Characterization Using 3D Gradation Curves" in CEUR Workshop Proceedings. Vol.1814 (2017) pp. 74-83.

12. O. Milder and D. Tarasov "Ink-jet printer's characterization by 3D gradation trajectories on an equidistant color difference basis". Lecture Notes in Computer Science, vol. 10749 (2018) pp. 40-52.

13. O. Milder and D. Tarasov "Gradation surfaces as a method for multi-color ink-jet printers color specifications management" in Lecture Notes in Computer Science (LNCS), vol. 10749 (2018) pp. 53-61.

14. D.A. Tarasov and O.B. Milder, Comp. Optics 43 (1), 132-136 (2019) (In Russian).
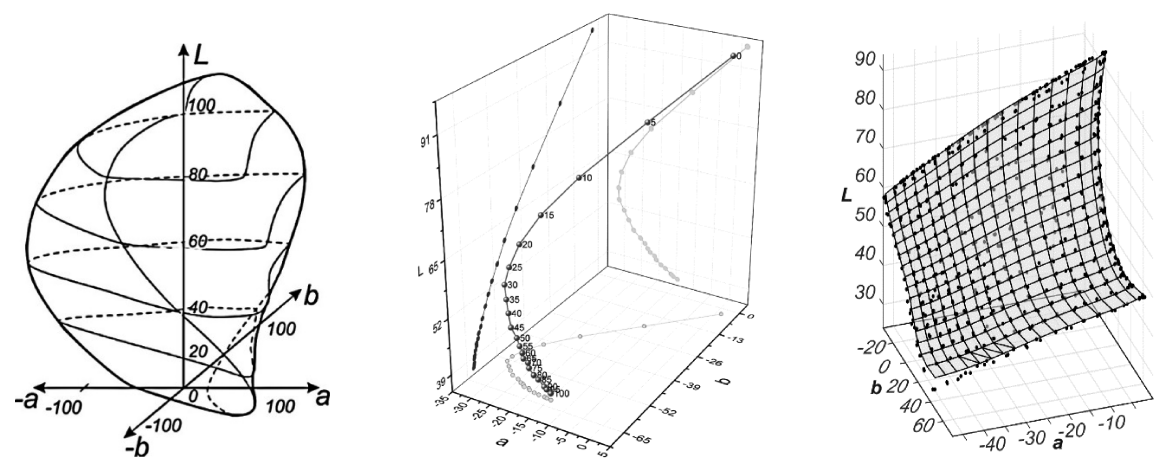


\begin{tabular}{|c|}
\hline Creating and printing the test scale \\
\hline$\downarrow$ \\
\hline Measuring Lab coordinates with a spectrophotometer \\
\hline$\downarrow$ \\
\hline $\begin{array}{l}\text { Sorting the measurement protocol data by binary color } \\
\text { channels }(R=M+Y, G=C+Y, B=C+M)\end{array}$ \\
\hline$\downarrow$ \\
\hline Approximation of the coordinates by functions $f(m, n)$ \\
\hline$\downarrow$ \\
\hline $\begin{array}{l}\text { Calculation of the color difference surface from the } \\
\text { substrate as a function of the recipes for both colorants } \\
\qquad d E_{2000}=f(\underline{m}, n)\end{array}$ \\
\hline$\downarrow$ \\
\hline Introduction of a fictitious parameter $p=m+n$ \\
\hline$\downarrow$ \\
\hline $\begin{array}{l}\text { Calculating the isolines } d E_{2000}(m, n)=f(p), p=\text { const } \\
\text { belonging to the surface } d E_{2000}=f(m, n)\end{array}$ \\
\hline$\downarrow$ \\
\hline $\begin{array}{l}\text { Defining the point on each isoline corresponding to the } \\
\text { minimum sum of color differences between the initial and } \\
\text { target points }\end{array}$ \\
\hline$\downarrow$ \\
\hline Computing geodesics in the parametric form \\
\hline
\end{tabular}

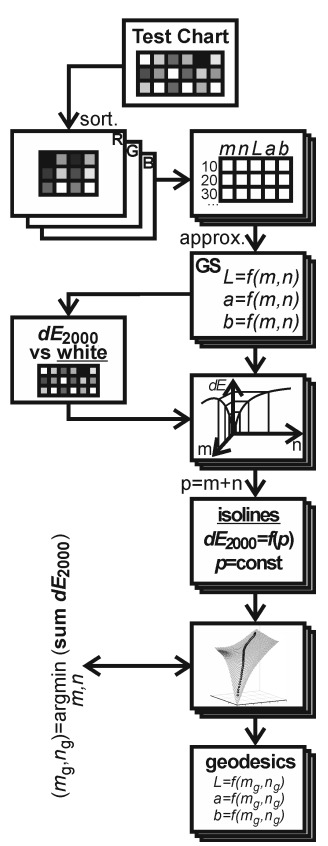




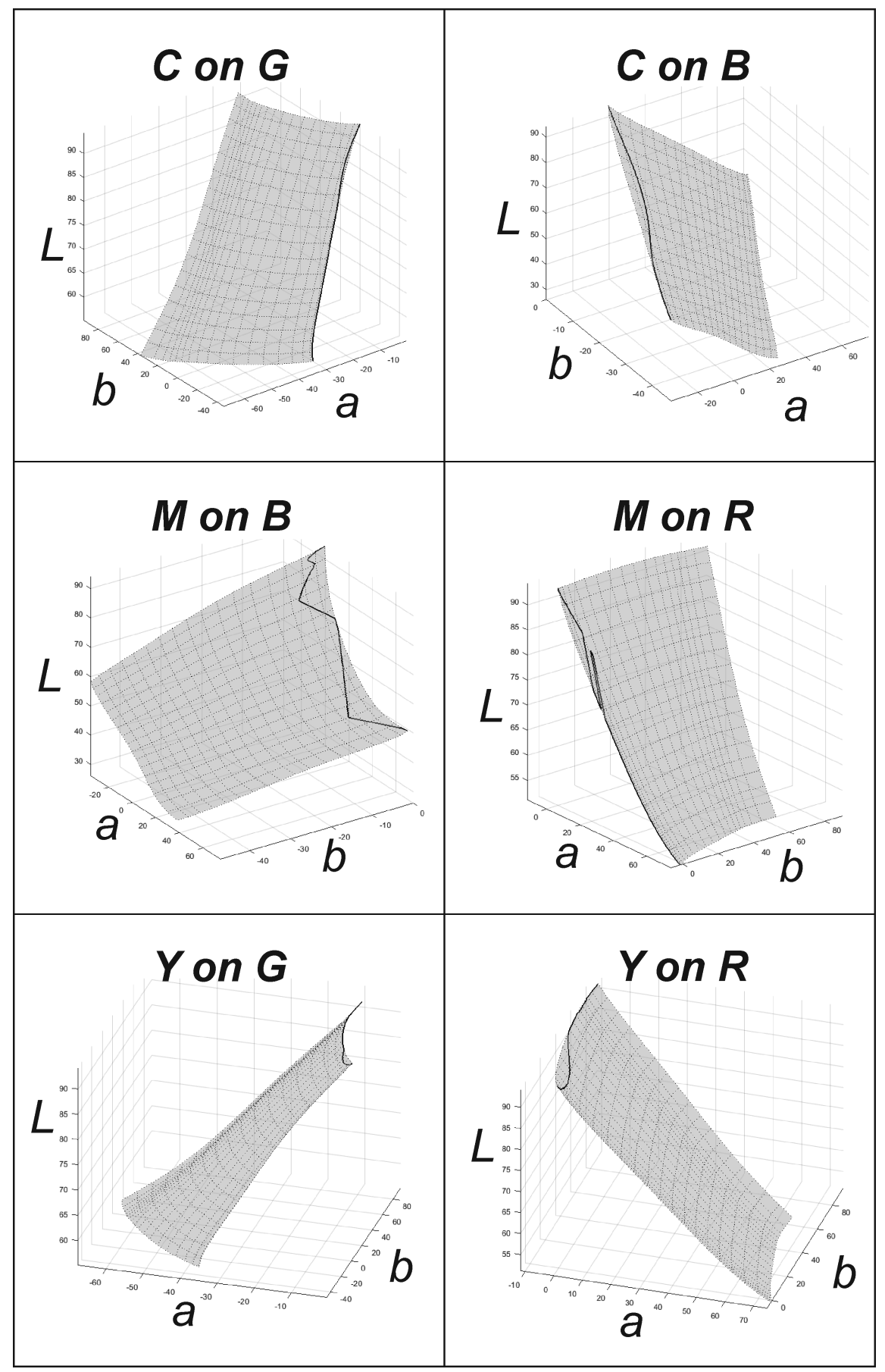

